\title{
artigo
}

\section{Não conformidades no processo de administração de antimicrobianos: revisão integrativa}

\author{
Non-conformities in the antimicrobial administration process: integrative review \\ No conformidades en el proceso de administración antimicrobiano: revisión integrativa
}

\begin{abstract}
RESUMO
Objetivo: identificar as possivveis não conformidades no processo de administração de antimicrobianos descritas em literatura. Método: revisão integrativa, com busca realizadas nas bases de dados Web of Science, PUBMED, LILACS e BDENF. A pergunta norteadora foi elaborada de acordo com a estratégia PICo: P (população) - enfermagem; I (interesse) - não conformidades; Co (contexto) - administração de antimicrobianos, sendo: Quais são as possíveis não conformidades no processo de administração de antimicrobianos descritas em literatura? Critérios de inclusão: artigos com resumos e textos completos nos idiomas português, inglês ou espanhol e publicados no período de 2014 a 2019. A busca foi realizada entre dezembro de 2019 e janeiro de 2020. Resultados: nove artigos foram selecionados para a pesquisa. As principais não conformidades apontadas foram erros de dose e atraso na administração de antimicrobianos. Todos os estudos foram do nível de evidência quatro. Conclusão: Sugere-se que o enfermeiro amplie suas ações nessa temática.
\end{abstract}

DESCRITORES: Gestão de Antimicrobianos; Erros de Medicação; Enfermagem; Anti-infecciosos; Vias de Administração de Medicamentos.

\section{ABSTRACT}

Objective: to identify the possible non-conformities in the antimicrobial administration process described in the literature. Method: integrative review, with searches carried out in the databases Web of Science, PUBMED, LILACS e BDENF. The guiding question was developed according to the PICo strategy: P (population) - nursing; I (interest) - non-conformities; Co (context) - antimicrobial administration, being: What are the possible non-conformities in the antimicrobial administration process described in the literature? Inclusion criteria: articles with abstracts and full texts in Portuguese, English or Spanish and published between 2014 and 2019. The search was carried out between December 2019 and January 2020. Results: nine articles were selected for the research. The main non-conformities pointed out were dose errors and delay in the administration of antimicrobials. All studies were at evidence level four. Conclusion: It is suggested that nurses expand their actions on this topic.

DESCRIPTORS: Antimicrobial Management; Medication Errors; Nursing; Anti-infectives; Drug Administration Routes.

\section{RESUMEN}

Objetivo: identificar las posibles no conformidades en el proceso de administración de antimicrobianos descritos en la literatura. Método: revisión integradora, con búsquedas realizadas en las bases de datos Web of Science, PUBMED, LILACS e BDENF. La pregunta guía se desarrolló de acuerdo con la estrategia PICo: P (población) - enfermería; I (interés) - no conformidades; Co (contexto) - administración de antimicrobianos, siendo: ¿Cuáles son las posibles no conformidades en el proceso de administración de antimicrobianos descritos en la literatura? Los criterios de inclusión: artículos con resúmenes y textos completos en portugués, inglés o español y publicados entre 2014 y 2019. La búsqueda se realizó entre diciembre de 2019 y enero de 2020. Resultados: nueve artículos se seleccionaron para la investigación. Las principales no conformidades señaladas fueron errores de dosis y retraso en la administración de antimicrobianos. Todos los estudios estaban en el nivel de evidencia cuatro. Conclusión: Se sugiere que las enfermeras amplíen sus acciones sobre este tema.

DESCRIPTORES: Manejo de antimicrobianos; Errores de medicación; Enfermería; Antiinfecciosos; Vías de Administración de Medicamentos.

RECEBIDO EM: 13/01/2021 APROVADO EM: 01/02/2021

\section{Daniela Sanches Couto}

Enfermeira, Mestranda em Ciências da Saúde, Universidade Federal de São Carlos- UFSCar.

ORCID: 0000-0003-0767-4000 


\section{Lívia Cristina Scalon da Costa Perinoti}

Enfermeira, Mestre em Enfermagem, Doutoranda em Ciências da Saúde, Universidade Federal de São Carlos- UFSCar. ORCID: 0000-0002-7056-8852

\section{Adriana Maria da Silva Felix}

Enfermeira, Doutora em Ciências, Professora do Departamento de Enfermagem em Saúde Coletiva da Faculdade de Ciências Médicas da Santa Casa de SP (FCMSCSP).

ORCID: 0000-0002-3559-3729

\section{Rosely Moralez de Figueiredo}

Enfermeira, Doutora em Saúde Mental, Professora Titular da Universidade Federal de São Carlos-UFSCar.

ORCID: 0000-0002-0131-4314

\section{INTRODUÇÃO}

A resistência antimicrobiana é a aptidão das bactérias sobreviverem aos antimicrobianos quando estas drogas deveriam inibir o crescimento ou destruir os microrganismos. Esse fato tem se intensificado devido ao seu uso indiscriminado, gerando gastos extras para os sistemas de saúde e reduzindo as alternativas terapêuticas ${ }^{1,2}$.

Para intervir nesse problema global a Organização Mundial de Saúde criou o Plano de Ação Global sobre Resistência Antimicrobiana, apresentando como principal estratégia o Programa de Gerenciamento do uso de Antimicrobiano ou Antimicrobial Stewardship ${ }^{2-4}$.

Ações que visem a administração de antimicrobiano em tempo oportuno estão diretamente relacionadas tanto com a redução da resistência microbiana como com a prevenção de erros de medicação.

A enfermagem desempenha papel crucial nas fases de preparo e administração de medicamentos sempre preocupandose com a segurança do paciente por meio do controle de horários, diluições e intervalos adequados. Cabe a estes profissionais desenvolver ações visando o aumento da segurança, minimização de erros e garantindo a eficácia do tratamento ${ }^{5-7}$.

O Conselho Regional de Enfermagem de São Paulo orienta aos seus profissionais o uso dos certos para a medicação segura: anotação correta, medicamento certo, via certa, hora certa, dose certa, paciente certo, orientação ao paciente, compatibilidade medicamentosa e direito de recusar o medicamento ${ }^{8}$.
A enfermagem desempenha papel crucial nas fases de preparo $\mathrm{e}$ administração de medicamentos sempre preocupando-se com a segurança do paciente por meio do controle de horários, diluições e intervalos adequados. Cabe a estes profissionais desenvolver ações visando o aumento da segurança, minimização de erros e garantindo a eficácia do tratamento
Sendo assim, é indispensável a ampliação do conhecimento a respeito dos fatores que interferem no processo de administração de antimicrobianos?

Esse estudo teve por objetivo identificar as possíveis não conformidades no processo de administração de antimicrobianos descritas em literatura.

\section{MÉTODOS}

A partir da Prática Baseada em Evidência realizou-se uma revisão integrativa em seis etapas: elaboração da pergunta norteadora, busca ou amostragem na literatura, coleta de dados, análise crítica dos estudos incluídos, discussão dos resultados e, por fim, apresentação dos resultados encontrados ${ }^{9,10}$.

O estudo foi norteado por protocolo desenvolvido pelos pesquisadores. A questão de pesquisa foi elaborada de acordo com a estratégia População Interesse Contexto (PICo) ${ }^{11}$, sendo: $\mathrm{P}$ (população) - enfermagem; I (interesse) - não conformidades; Co (contexto) - administração de antimicrobianos. Assim, elaborou-se a seguinte questão norteadora: "Quais são as possíveis não conformidades no processo de administração de antimicrobianos descritas em literatura? " Feito isso, realizou-se a etapa de busca entre dezembro de 2019 e janeiro de 2020. Critérios de inclusão: artigos com resumos e textos completos nos idiomas português, inglês ou espanhol, publicados no período de janeiro de 2014 a dezembro de 2019, considerando que a temática é atual e o Plano de Ação Global sobre Resistência Antimicrobiana2 foi publicado em 2015. Já os critérios 


\section{artigo}

Couto, D.S.; Perinoti, L.C.S.C.; Felix, A.M.S.; Figueiredo, R.M.;

Não conformidades no processo de administração de antimicrobianos: revisão integrativa

de exclusão: teses, dissertações, estudo-piloto, artigos de revisão, cartas, editorais e resumos de eventos.

Foram utilizadas as seguintes bases de dados: Web of Science, Biblioteca Nacional de Medicina dos Estados Unidos (PUBMED), Latin American and Caribbean Health Science Literature Database (LILACS) e Base de Dados de Enfermagem (BDENF). Optou-se por utilizar essas bases por contemplarem os principais periódicos da área da saúde e da enfermagem, que tratam do tema de interesse do presente estudo.

Fez-se uso dos Descritores em Ciências da Saúde (DeCS) e Medical Subject Hea$\operatorname{ding}(\mathrm{MeSH})$ : Antimicrobial Stewardship
(Gestão de Antimicrobianos, Programas de Optimización del Uso de los Antimicrobianos); Medication Errors (Erros de Medicação, Errores de Medicación); Nursing (Enfermagem, Enfermería); Anti-Infective Agents (Anti-Infecciosos, Antiinfecciosos). Utilizou-se o operador booleano and e associações entre todos os descritores em português, inglês e espanhol, exceto nas bases PUBMED e Web of Science que se apresentam apenas no idioma inglês.

Foi realizada a classificação dos níveis de evidência dos artigos selecionados para a pesquisa ${ }^{12}$ : nível 1 - metanálise de estudos clínicos controlados randomizados; nível 2- estudo de desenho experimental; nível
Figura 1 - Fluxograma dos artigos localizados, lidos título e resumo, selecionados para leitura na íntegra, excluídos, duplicados e selecionados para a pesquisa. São Carlos, SP, 2020.

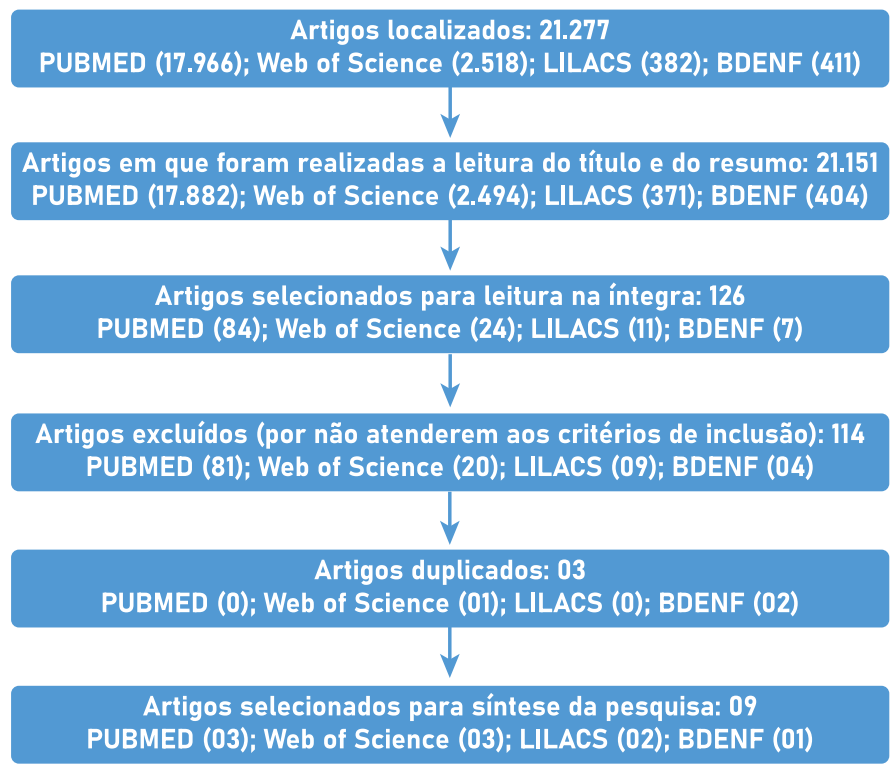

3- delineamento de estudo quase-experimental; nível 4- estudos não experimentais, descritivos ou com abordagem metodológica qualitativa ou estudo de caso; nível 5 - relatório de casos ou dado obtido de forma sistemática, de qualidade verificável ou dados de avaliação de programas; nível 6 - opinião de especialistas, baseadas nas experiências clínicas ou de comitê de especialistas, incluindo interpretações de informações não baseadas em pesquisas, em opiniões regulares ou legais. A extração dos dados foi baseada em instrumento validado $^{13}$. Os resultados foram apresentados de forma descritiva.

\section{RESULTADOS}

Dentre os 21.277 artigos localizados, nove artigos atenderam aos critérios de inclusão, conforme apresentado na Figura 1.

Quanto aos países de realização dos estudos, quatro deles foram desenvolvidos no Brasil (44,44\%). O Malawi publicou dois artigos (22,23\%). Já Canada, Índia e Austrália contaram com apenas um estudo em cada país (11,11\% cada). Em se tratando do idioma em que o assunto foi mais publicado, destaca-se o Inglês com seis pesquisas $(66,67 \%)$, seguido do português, com três (33,33\%). Embora descritores em espanhol tenham sido utilizados, não foi localizado nenhum artigo nesse idioma.

Todos os estudos incluídos foram classificados em nível de evidência quatro (estudos não experimentais, descritivos ou com abordagem metodológica qualitativa ou estudo de caso) $)^{12}$.

O Quadro 1 traz a síntese das análises dos artigos selecionados para a pesquisa e seus principais resultados.

\section{Quadro 1 - Caracterização dos artigos publicados sobre as não-conformidades no processo de administração de} antimicrobianos. São Carlos, SP, Brasil, 202013

\begin{tabular}{|l|c|c|c|}
\hline AUTOR & OBJETIVO & TIPO DE ESTUDO & PRINCIPAIS RESULTADOS \\
\hline $\begin{array}{l}\text { Pereira et al., } \\
2018 \mathrm{a}^{14}\end{array}$ & $\begin{array}{c}\text { Identificar interações medi- } \\
\text { camentosas induzidas pelo } \\
\text { aprazamento e os erros no } \\
\text { preparo de antibacterianos } \\
\text { administrados. }\end{array}$ & $\begin{array}{c}\text { Observacional e } \\
\text { transversal }\end{array}$ & $\begin{array}{c}\text { Em 81 observações (32,5\%) houveram erros de dose. O tempo de } \\
\text { preparo apresentou-se prolongado em algumas situações. }\end{array}$ \\
\hline
\end{tabular}




\begin{tabular}{|c|c|c|c|}
\hline $\begin{array}{l}\text { Santos et al., } \\
2016^{15}\end{array}$ & $\begin{array}{l}\text { Identificar fatores de risco } \\
\text { na administração de anti- } \\
\text { microbianos pela equipe de } \\
\text { enfermagem. }\end{array}$ & $\begin{array}{l}\text { Estudo retrospec- } \\
\text { tivo, descritivo, de } \\
\text { análise documental }\end{array}$ & $\begin{array}{l}\text { Foram identificados: falta de anotações sobre sinais flogísticos; } \\
\text { inadequações nos horários de administração dos antimicrobia- } \\
\text { nos; falta de adoção de medidas de precaução e isolamento e de } \\
\text { coletas de swabs. }\end{array}$ \\
\hline $\begin{array}{l}\text { Mula et al., } \\
2019^{16}\end{array}$ & $\begin{array}{l}\text { Examinar comportamentos } \\
\text { que enfermeiros e médicos } \\
\text { empregam para enfrentar } \\
\text { os desafios encontrados } \\
\text { durante a administração de } \\
\text { antibióticos. }\end{array}$ & $\begin{array}{l}\text { Estudo de caso } \\
\text { qualitativo, des- } \\
\text { critivo }\end{array}$ & $\begin{array}{l}\text { As práticas foram elencadas em dois grupos: "Técnicas que } \\
\text { alteram o procedimento" (diluição incorreta, preparo de diversas } \\
\text { doses antes do horário de uso e falta de horário correto da admi- } \\
\text { nistração, mencionando apenas manhã, tarde e noite) e "Uso de } \\
\text { procedimentos não autorizados" (alteração de dose, checagem } \\
\text { e preparo muito antes da administração, prescrição médica para } \\
\text { melhor atender aos enfermeiros). }\end{array}$ \\
\hline $\begin{array}{l}\text { Pereira et al., } \\
2016^{7}\end{array}$ & $\begin{array}{l}\text { Avaliar as conformidades } \\
\text { e não conformidades no } \\
\text { preparo e administração de } \\
\quad \text { antibacterianos. }\end{array}$ & $\begin{array}{l}\text { Observacional e } \\
\text { transversal }\end{array}$ & $\begin{array}{c}\text { Houve, em todas as observaçães, descumprimento dos preceitos } \\
\text { da semiotécnica, evidenciado por: ausência de desinfecção das } \\
\text { ampolas do medicamento, não utilização de luvas durante o } \\
\text { procedimento, respingos de antibacteriano dispersados no ar, } \\
\text { contaminaçãa do êmbolo das seringas, e preparo com antece- } \\
\text { dência superior a } 30 \text { minutos do momento da administração. } \\
\text { Os itens de checagem do medicamento administrado, monito- } \\
\text { ramento e controle do tempo de infusão foram mais incidentes } \\
\text { para não-conformidades, com } 172(64,9 \%), 166(62,7 \%) \text { e } 159 \\
(60 \%) \text {, respectivamente. }\end{array}$ \\
\hline $\begin{array}{l}\text { Mula et al., } \\
2018^{17}\end{array}$ & $\begin{array}{l}\text { Identificar fatores que } \\
\text { influenciam o início opor- } \\
\text { tuno de antibióticos após } \\
\text { a prescrição no Centro de } \\
\text { Emergência e Trauma de } \\
\text { Adultos de um hospital. }\end{array}$ & Estudo de Caso & $\begin{array}{l}\text { Os pacientes não receberam a primeira dose de antibiótico } \\
\text { dentro de uma hora após a prescrição. As possíveis barreiras para } \\
\text { o início oportuno dos antibióticos foram: longas esperas, falta de } \\
\text { comunicação / atendimento coordenado e falta de competência. }\end{array}$ \\
\hline $\begin{array}{l}\text { Pereira et al., } \\
\text { 2018b }{ }^{18}\end{array}$ & $\begin{array}{l}\text { Identificar a relação entre } \\
\text { os fatores ambientais e os } \\
\text { erros de preparo e adminis- } \\
\text { tração de medicamentos } \\
\text { antibacterianos. }\end{array}$ & $\begin{array}{l}\text { Observacional e } \\
\text { transversal }\end{array}$ & $\begin{array}{l}\text { As principais categorias de erros encontradas foram: erro de dose } \\
\text { (157), erro de horário (30) e escolha errada do medicamento (28). }\end{array}$ \\
\hline $\begin{array}{l}\text { Chambers et al., } \\
2019^{19}\end{array}$ & $\begin{array}{l}\text { Descrever as fontes de } \\
\text { dados e os processos } \\
\text { usados para desenvolver } \\
\text { um programa para reduzir o } \\
\text { uso excessivo de antibióti- } \\
\text { cos nos cuidados de longo } \\
\text { prazo. }\end{array}$ & Survey & $\begin{array}{l}\text { A avaliação de barreiras/ facilitadores mostrou necessidade de } \\
\text { uma abordagem com a inclusão de estratégias (1) para estabe- } \\
\text { lecer adesão às mudanças; (2) alinhar políticas e procedimentos } \\
\text { organizacionais; (3) fornecer educação e apoio contínuo ao trei- } \\
\text { namento de funcionários; (4) fornecer informações e educação a } \\
\text { residentes e familias; (5) estabelecer vigilância do processo com } \\
\text { feedback para a equipe; (6) para enviar lembretes. }\end{array}$ \\
\hline $\begin{array}{l}\text { James et al., } \\
2015^{20}\end{array}$ & $\begin{array}{l}\text { Obter uma compreensão } \\
\text { dos fatores que impactam } \\
\text { a Antimicrobial stewardship } \\
\text { em hospitais regionais e } \\
\quad \text { rurais da Austrália }\end{array}$ & Estudo qualitativo & $\begin{array}{l}\text { As principais barreiras percebidas foram falta de acesso à edu- } \\
\text { cação, recursos e apoio especializado. Os facilitadores eram uma } \\
\text { estrutura de governança mais plana, maior senso de orgulho, } \\
\text { desejo de sucesso e bom acesso à Internet e telessaúde. }\end{array}$ \\
\hline $\begin{array}{l}\text { Baubie et al., } \\
2019^{21}\end{array}$ & $\begin{array}{l}\text { Determinar quais barreiras } \\
\text { e facilitadores à administra- } \\
\text { ção de antibióticos existem } \\
\text { em um estabelecimento de } \\
\text { saúde. }\end{array}$ & Estudo qualitativo & $\begin{array}{l}\text { Identificou-se as seguintes barreiras: acesso limitado a farma- } \\
\text { cêuticos clínicos, oposição do médico a mudanças em relação às } \\
\text { políticas de administração, diminuição frequente de antibióticos, } \\
\text { alta carga de trabalho do médico, registro médico eletrônico } \\
\text { incompleto, visibilidade inadequada do Stewardship e alto nível } \\
\text { de uso de antibióticos na comunidade. }\end{array}$ \\
\hline
\end{tabular}




\section{artigo}

Couto, D.S.; Perinoti, L.C.S.C.; Felix, A.M.S.; Figueiredo, R.M.i

Não conformidades no processo de administração de antimicrobianos: revisão integrativa

\section{DISCUSSÃO}

As principais não conformidades identificadas foram doses errôneas e atrasos na administração de antimicrobianos ${ }^{14-18}$.

A resistência antimicrobiana ocasiona o surgimento de infecções praticamente intratáveis, sendo esse problema uma das maiores ameaças recentes à saúde pública. Esse fato se acentua com a falta de pesquisas na área e até mesmo com práticas inadequadas dos profissionais de saúde envolvidos neste processo $^{22,23}$, dentre eles, a equipe de enfermagem.

Corroborando com os achados da atual pesquisa, estudo realizado por MANZO et al. ${ }^{5}$, que objetivou investigar a prática dos profissionais de enfermagem sobre o processo de administração de medicamentos em geral e as circunstâncias que levam aos erros, relatou que cerca de $61 \%$ das administrações foram realizadas com dose incorreta e $21 \%$ em horário incorreto.

Outro estudo, uma revisão integrati$\mathrm{va}^{24}$, que teve como objetivo identificar as evidências e as implicações dos erros na administração de medicação em geral na segurança do paciente, encontrou dados semelhantes a esta revisão, sendo que, de um total de 40 artigos, $67,5 \%$ traziam o erro de dosagem e $50 \%$ erro de horário.

Atraso de administração de medicações são sempre prejudiciais, entretanto, se tratando de antimicrobianos este atraso pode ter consequências ainda mais graves e até mesmo irreversíveis, além do aumento da resistência antimicrobiana. Em casos de pacientes sépticos, por exemplo, a precisão no início e manutenção da terapia antimicrobiana está diretamente relacionada com a taxa de sobrevivência do paciente ${ }^{25}$.

Devido ao importante papel da equipe de enfermagem nesse processo, garantindo a terapêutica, promovendo a segurança do paciente e colaborando para se evitar a resistência antimicrobiana, é fundamental conhecimento adequado para esse $\mathrm{fim}^{26}$.

$O$ enfermeiro tem um papel importante na gestão de antimicrobianos (Antimicrobial Stewardship), tanto na minimização dos fatores que interferem na administração antimicrobiana e consequentemente, na resistência antimicrobiana, como na detecção precoce de sinais de infecção e orientação para otimização do tratamento antimicrobiano ${ }^{27}$. Porém, ainda há necessidade de ampliação do ensino sobre o tema para que a estratégia seja efetiva ${ }^{28-30}$. Ressalta-se a falta de educação permanente ${ }^{19,20}$, sendo esta uma ação que auxiliaria no enfrentamento das principais não conformidades encontradas ${ }^{14-18}$.

A importância de ampliar as pesquisas nessa temática também pode ser vista no reduzido número de estudos selecionados e no tipo de delineamento metodológico (nível de evidência IV) identificados em todos os estudos desta revisão integrativa.

As limitações deste estudo residem na dificuldade de se generalizar os resultados obtidos devido à carência da existência de investigações com maior rigor metodológico.

\section{CONCLUSÃO}

Concluiu-se que as principais não conformidades no processo de administração de antimicrobianos apontados em literatura foram erros de doses e atrasos na administração.

Sugere-se que o enfermeiro, peça-chave na gestão de antimicrobianos amplie suas ações nessa temática, tanto em atividades de educação permanente como seu engajamento em pesquisas visando a incorporação de resultados na prática clínica com consequente contribuição na redução da resistência antimicrobiana.

\section{REFERÊNCIAS}

1. Courtenay M, Castro-Sánchez E. Antimicrobial Stewardship for Nurses. JAC-Antimicrobial Resist. 2019;1:1-10.

2. World Health Organization. Global Action Plan on Antimicrobial Resistance. World Heal Organ [Internet]. 2015;1-28. Available from: http://www.emro.who.int/health-topics/drug-resistance/ global-action-plan.html

3. Grabe MJ, Resman F. Antimicrobial Stewardship: What We All Just Need to Know. Eur Urol Focus [Internet]. 2019;5:46-9. Available from: https://doi.org/10.1016/j.euf.2018.06.012

4. Laks M, Guerra CM, Miraglia JL, Medeiros EA. Distance learning in antimicrobial stewardship: Innovation in medical education. BMC Med Educ [Internet]. 2019;19:1-9. Available from: https://doi.org/10.1186/s12909-019-1623-x

5. Manzo BF, Brasil CLGB, Reis FFT, Correa A dos R, Simão DA da S, Costa ACL. Seguridad en la administración de medicamentos: investigación sobre la práctica de enfermería y circunstancias de errores. Enferm Glob [Internet]. 2019;18:19-31. Available from:
http://dx.doi.org/10.6018/eglobal.18.4.344881\%OA

6. Brasil. PROTOCOLO DE SEGURANÇA NA PRESCRIÇÃO, USO E ADMINISTRAÇÃO DE MEDICAMENTOS. Ministério da Saúde [Internet]. 2013; Available from: https://www20.anvisa.gov.br/ segurancadopaciente/index.php/publicacoes/item/seguranca-na-prescricao-uso-e-administracao-de-medicamentos

7. Pereira FGF, Aquino GÂ de, Melo GAA, Praxedes CDO, Caetano JÁ. CONFORMIDADES E NÃO CONFORMIDADES NO PREPARO E ADMINISTRAÇÃO DE ANTIBACTERIANOS. Cogitare Enferm. 2016;21:1-9.

8. Conselho Regional de Enfermagem. Uso seguro de medicamentos: Guia de preparo, administração, monitoramento. São Paulo COREN. 2017

9. Mendes KDS, Silveira RC de CP, Galvão CM. Revisão integrativa: método de pesquisa para a incorporação de evidências na saúde e na enfermagem. Texto Context - Enferm. 2008;17:758_64. 


\section{REFERÊNCIAS}

10. Souza MT de, Silva MD da, Carvalho R de. Revisão integrativa: o que é e como fazer. Reme Rev Min Enferm [Internet]. 2010;8:102-6. Available from: http://www.scielo.br/ pdf/rlae/v12n3/v12n3a14\%OAhttp://www.scielo.br/scielo. php?script=sci_arttext\&pid=S0102-311X2007000400002\&Ing=pt\&tlng=pt\%OAhttp://www.scielo.br/scielo.php?script=sci_arttext\&pid=S0104-07072008000400018\&Ing=pt\&tIng=pt\%OAhttp://www.ncbi.

11. Lockwood C, Porritt K, Munn Z, Rittenmeyer L, Salmond S, Bjerrum $M$, et al. Chapter 2 : Systematic reviews of qualitative evidenceln: Aromataris E, Munn Z (Editors). Joanna Briggs Institute Reviewer's Manual. [Internet]. The Joanna Briggs Institute. 2017. Available from: https://reviewersmanual.joannabriggs. org/

12. Stetler CB, Morsi D, Rucki S, Broughton S, Corrigan B, Fitzgerald J, et al. Utilization-Focused Integrative Reviews in a Nursing Service. Clin Methods [Internet]. 1998;11:195-206. Available from: https://www.sciencedirect.com/science/article/pii/ S0897189798803297?via\%3Dihub

13. Ursi ES, Gavão CM. Prevenção de lesões de pele no perioperatório: Revisão integrativa da literatura. Rev Lat Am Enfermagem [Internet]. 2006;14:124-31. Available from: http://www. scielo.br/scielo.php?script=sci_abstract\&pid=S0104-1169200 6000100017\&tlng=pt

14. Pereira FGF, Melo GAA, Galindo Neto NM, Carvalho REFL de, Néri EDR, Caetano JÁ. Interações medicamentosas induzidas pelo aprazamento e os erros no preparo de antibacterianos. Rev da Rede Enferm do Nord. 2018;19:3322.

15. Santos RC dos, Pessalacia JDR, Mata LRF da. Risk factors in the management of antimicrobial agents in nursing. Acta Sci Heal Sci [Internet]. 2016;38:49-55. Available from: https://doi. org/10.4025/actascihealthsci.v38i1.28505

16. Mula CT, Human N, Middleton L. An exploration of workarounds and their perceived impact on antibiotic stewardship in the adult medical wards of a referral hospital in Malawi: A qualitative study. BMC Health Serv Res [Internet]. 2019;19:1-10. Available from: https://doi.org/10.1186/s12913-019-3900-0

17. Mula CT, Middleton L, Human N, Varga C. Assessment of factors that influence timely administration of initial antibiotic dose using collaborative process mapping at a referral hospital in Malawi: A case study of pneumonia patients. BMC Infect Dis [Internet]. 2018;18:1-13. Available from: http://www.embase. com/search/results? subaction=viewrecord\&from=export\&id=L625710617\%OAhttp://dx.doi.org/10.1186/s12879018-3620-9

18. Pereira FGF, Ataíde MBC de, Silva RL, Néri EDR, Carvalho GCN, Caetano JÁ. Environmental variables and errors in the preparation and administration of medicines. Rev Bras Enferm [Internet]. 2018;71:1046-54. Available from: https://pesquisa. bvsalud.org/portal/resource/pt/biblio-958622

19. Chambers A, Macfarlane S, Zvonar R, Evans G, Moore JE, Langford $B J$, et al. A recipe for antimicrobial stewardship success: Using intervention mapping to develop a program to reduce an- tibiotic overuse in long-term care. Infect Control Hosp Epidemiol [Internet]. 2019;40:24-31. Available from: doi:10.1017/ ice.2018.281

20. James R, Luu S, Avent M, Marshall C, Thursky K, Buising K. A mixed methods study of the barriers and enablers in implementing antimicrobial stewardship programmes in Australian regional and rural hospitals. J Antimicrob Chemother [Internet] 2015;70:2665-70. Available from: doi:10.1093/jac/dkv159

21. Baubie K, Shaughnessy C, Kostiuk L, Varsha Joseph M, Safdar $\mathrm{N}$, Singh SK, et al. Evaluating antibiotic stewardship in a tertiary care hospital in Kerala, India: A qualitative interview study. BMJ Open [Internet]. 2019;9:1-7. Available from: https://bmjopen. bmj.com/content/9/5/e026193

22. Mason T, Trochez C, Thomas R, Babar M, Hesso I, Kayyali R. Knowledge and awareness of the general public and perception of pharmacists about antibiotic resistance. BMC Public Health [Internet]. 2018;18:1-10. Available from: https://doi. org/10.1186/s12889-018-5614-3

23. Munita JM, Arias CA. Mechanisms of Antibiotic Resistance. HHS Public Access [Internet]. 2016;4:1-37. Available from: doi:10.1128/microbiolspec.VMBF-0016-2015

24. Gomes AT de L, Salvador PTC de O, Rodrigues CCFM, Silva M da F, Ferreira $L$ de $L$, Santos VEP. Patient safety in nursing paths in Brazil. Rev Bras Enferm [Internet]. 2017;70:139-46. Available from: http://dx.doi.org/10.1590/0034-7167-2015-0139

25. Rêgo HCLJ, Lima KVB, Xavier MB. Antibioticoterapia e sobrevivência de pacientes sépticos em hospital de alta complexidade, Belém/PA. Enferm Bras [Internet]. 2019;18:220-4. Available from: https://doi.org/10.33233/eb.v18i2.2116

26. Fassarella CS, Bueno AAB, Souza ECC de. SEGURANÇA DO NO AMBIENTE HOSPITALAR: OS AVANÇOS NA PREVENÇÃ̂O DE EVENTOS ADVERSOS NO SISTEMA DE MEDICAÇÃO. Rev Rede Cuid em Saúde [Internet]. 2013;7:1-8. Available from: http:// publicacoes.unigranrio.edu.br/index.php/rcs/article/view/1897

27. Padoveze MC, Abraão LM, Figueiredo RM. Antimicrobials and antimicrobial resistance. In: Antimicrobial Stewardship for Nurses. 2020. p. 25.

28. Carter EJ, Greendyke WG, Furuya EY, Srinivasan A, Shelley AN, Bothra $A$, et al. Exploring the nurses' role in antibiotic stewardship: A multisite qualitative study of nurses and infection preventionists. Am J Infect Control [Internet]. 2018;46:492-7. Available from: doi:10.1016/j.ajic.2017.12.016

29. Courtenay M, Lim R, Castro-Sanchez E, Deslandes R, Hodson K, Morris G, et al. Development of consensus-based national antimicrobial stewardship competencies for UK undergraduate healthcare professional education. J Hosp Infect [Internet] 2018;100:245-56. Available from: https://doi.org/10.1016/j. jhin.2018.06.022

30. Felix AM da S, Toffolo SR. O enfermeiro nos programas de gerenciamento do uso de antimicrobianos: revisão integrativa. Cogitare Enferm [Internet]. 2019;24. Available from: http://dx. doi.org/10.5380/ce.v24i0.59324 